\title{
Editorial: Topical Collection on Jets and Winds in Pulsar Wind Nebulae, Gamma-Ray Bursts and Blazars
}

\section{Physics of Extreme Energy Release}

\author{
A. Bykov ${ }^{1,2,3}$ - E. Amato ${ }^{4,5}$ - J. Arons ${ }^{6}$ - M. Falanga ${ }^{3}$. \\ M. Lemoine ${ }^{7} \cdot$ L. Stella ${ }^{8} \cdot$ R. von Steiger $^{3}$
}

Received: 25 April 2017 / Accepted: 2 May 2017 / Published online: 12 June 2017

(C) Springer Science+Business Media Dordrecht 2017

The ever increasing sensitivity and improving accuracy of multi-wavelength observations have offered in recent decades an unprecedented insight into the physics of relativistic astrophysical sources, most notably, gamma-ray bursts, active galactic nuclei, microquasars, and pulsar wind nebulae. The superb angular resolution images of pulsar wind nebulae and extragalactic jets provided by the modern radio interferometers, the Hubble Space Telescope and Chandra X-ray observatory, as well as time resolved spectra obtained with modern gamma-ray telescopes are beautiful examples of these achievements. They allow us to reveal the intimate details of synchrotron nebulae and jets, which result from dissipation of highly relativistic magnetized winds originating in the central engines of such objects.

Relativistic objects in astrophysics present a diverse set of phenomena, cover vast ranges of time scales and spatial scales, but their descriptions rely on a similar backbone: production of a relativistic outflow in a central compact object and dissipation of the outflow at large radii accompanied by acceleration of particles up to very high energies and production of secondary non-thermal electromagnetic radiation. Studies in this field thus tackle a

\footnotetext{
A. Bykov

byk@astro.ioffe.ru

1 Ioffe Institute, 194021, St. Petersburg, Russia

2 St. Petersburg Politechnical University, St. Petersburg, Russia

3 International Space Science Institute, Bern, Switzerland

4 INAF Osservatorio Astrofisico di Arcetri, Largo E. Fermi 5, 50125 Firenze, Italy

5 Dipartimento di Fisica ed Astronomia, Universit‘ a degli Studi di Firenze, Via G. Sansone 1, 50019 Sesto Fiorentino, Firenze, Italy

6 Astronomy Department and Theoretical Astrophysics Center, University of California, Berkeley, 501 Campbell Hall, Berkeley, CA 94720, USA

7 Institut d'Astrophysique de Paris, CNRS-UPMC, 98 bis boulevard Arago, 75014 Paris, France

8 INAF-Osservatorio Astronomico di Roma, via Frascati 33, 00078, Monteporzio Catone, Roma, Italy
} 
number of fundamental physical problems, such as: how the gravitational, rotational and electro-magnetic energies of the central engine are converted into the observed radiation, how magneto-hydrodynamic flows are launched and pushed to relativistic velocities, how the energy of a bulk flow is transferred to a minority of ultra-relativistic particles, and so on. Such astrophysical objects also harbour matter and radiation at extreme physical conditions in terms of electro-magnetic field strengths, matter densities, flow velocities, or particle energies, which lie well beyond the reach of any terrestrial laboratory, thus providing us with a unique opportunity to test the fundamental laws of physics.

This volume is opened with the review by G. Romero et al., who present a broad discussion of the physics of relativistic jets on large spatial scales, found in active galactic nuclei and in microquasars.

Studies of gamma-ray bursts (GRBs) have become a hot and rapidly advancing field of today's astrophysics as soon as their cosmological origin was established about twenty years ago. Multi-wavelength observations of GRBs and some other related transients, including ultra-long bursts and tidal disruption events, are discussed in detail by R. Willingale and P. Mészáros in a dedicated review. GRB production by relativistic jets that start in a highly opaque zone near the central source is discussed by A.M. Beloborodov and P. Mészáros, who provide a theoretical description of photospheric emission models of the prompt phase of GRBs. They discuss dissipative and radiative processes in the jet and the radiation transfer mechanisms, which shape the observed nonthermal emission spectrum released from the GRB photosphere.

Pulsars emit relativistic outflows in a variety of forms. Their magnetized outflows consist of some combination of highly relativistic $\mathrm{e}^{ \pm}$pairs and, perhaps, ions. Nearby pulsar wind nebulae produced by the Crab, Vela and Geminga pulsars allowed us to perform detailed imaging observations of jets, torii and different types of fine emitting structures of synchrotron origin, being excellent laboratories for studies of the complex structure formation and particle acceleration in relativistic outflows. The physics of pulsar wind nebulae is discussed in a number of comprehensive reviews. The paper by B. Cerutti and A.M. Beloborodov presents a detailed review of the basic physical processes taking place in pulsar magnetospheres, at the base of the wind outflow, and of current efforts in the kinetic numerical simulation of these complex phenomena. It is followed by the review of O. Porth et al., who provide a detailed discussion of the morphology of pulsar wind nebulae and its numerical simulation in relativistic magneto-hydrodynamics. This discussion is nicely complemented by the review of S.P. Reynolds et al. on the observational properties of pulsar wind nebulae and magnetars in various wavelength domains. Then, the review paper by A.M. Bykov, E. Amato et al. offers an exhaustive discussion of the physics governing the so-called bow shock pulsar wind nebulae. Numerical modeling, radiative signatures and a possible origin of the fascinating X-ray structures observed in the Vela, Geminga, Guitar and the Lighthouse nebulae are discussed within the bow shock scenario. Pulsars and their wind nebulae are producing electron-positron outflows and therefore are considered as possible contributors to the enhanced cosmic ray positron fraction observed in PAMELA and AMS-02 space experiments-the issue also addressed in this review.

Fast energy release in the central engines producing relativistic outflows of different magnetization and particle acceleration capability is the key physical process at the core of the modeling of non-thermal spectra of astrophysical sources. Two reviews are therefore dedicated to the physics of fast energy release in relativistic astrophysical objects. The paper by R. Blandford et al. discusses magnetoluminescence - an important concept of a rapid energy release in catastrophic reconnection events, a scenario which is strongly motivated by the recent discovery of rapid variability and flares of $\mathrm{GeV}$ range radiation in the Crab nebula and 
in some blazars. Relativistic collisionless shock waves, which form at the interface between relativistic outflows and the external medium, have long been thought to give rise to an efficient Fermi process of particle acceleration. The underlying physical principles together with current efforts at their numerical modeling employing state of the art particle-in-cell and Monte Carlo techniques, are discussed in some detail by G. Pelletier et al.

Overall, this volume provides a timely and comprehensive review of the main physical processes involved in formation and dissipation of relativistic outflows. It will be particularly useful to postgraduate students and researchers active in various areas of astrophysics and space science.

This volume is based on an international workshop which took place at ISSI Bern from November 16 to November 20, 2015, gathering about forty leading experts in the physics of high-energy astrophysical objects. 